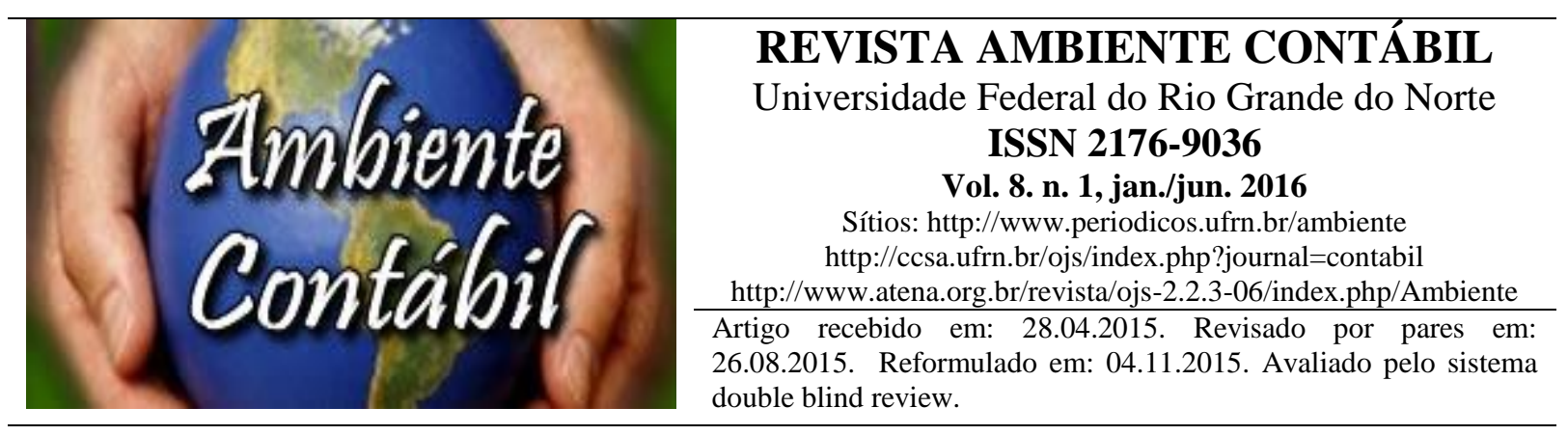

\title{
INTELIGÊNCIA COMPETITIVA: UM ESTUDO DO ENVOLVIMENTO DA ALTA DIREÇÃO NAS EMPRESAS DA REDEPETRO
}

\section{COMPETITIVE INTELLIGENCE: A STUDY OF THE INVOLVEMENT BY SENIOR CORPORATE MANAGEMENT AT REDEPETRO COMPANIES}

\section{INTELIGENCIA COMPETITIVA: UN ESTUDIO DEL ENVOLVIMIENTO DE LA ALTA DIRECCIÓN EN LAS EMPRESAS DE LA REDEPETRO}

Autores

\section{Tiago Henrique de Souza Echternacht}

Mestre em Ciências Contábeis pelo Programa Multiinstitucional e Inter-Regional UnB/UFPB/UFRN - Professor Assistente da Universidade Federal da Paraíba, Centro de Ciências Sociais Aplicadas - Campus I, Departamento de Finanças e Contabilidade. Endereço: Cidade Universitária - Castelo Branco - CEP: 58000-000 - Joao Pessoa, PB - Brasil Telefone: (83) 32167459 - Fax: (83) 32167459

Email:tiagoechternacht@gmail.com

\section{José Sueldo Câmara Ferreira}

Mestre em Administração pela UnP/ Laureate International Universities (2008) - Professor Assistente da Universidade do Estado do Rio Grande do Norte - UERN - Departamento: DCC - Departamento de Ciências Contábeis. Endereço: Av. Deputado Antônio Florêncio de Queiroz, 2995 - AP - 405-A Ponta Negra - CEP: 59092500

Natal - RN - Brasil - Telefone: (84) 9411-6268 - Fax: (84) 3317-4634

Email: sueldocamara@globo.com

\section{José Anízio Rocha de Araújo}

Doutor em Administração pela Pontifícia Universidade Católica do Paraná- PUC/PRProfessor Adjunto da Universidade Federal Rural do Semí Árido - UFERSA, Departamento: DCETH, Campus Angicos. Endereço: Rua: Gamaliel Martins Bezerra, s/n. Alto da Alegria, Angicos - RN, Brasil, CEP. 59.515-000, Telefone: (84) 3531-2547

Email: anizioaraujo@gmail.com

\section{Carlos Olavo Quandt}

Ph.D. em Urban Planning - University Of California Los Angeles (1993). Professor titular dos Programas de Graduação, Mestrado e Doutorado em Administração (PPAD) e Mestrado Profissional em Gestão de Cooperativas (PPGCOOP) da Pontifícia Universidade Católica do Paraná. Pontifícia Universidade Católica do Paraná, Centro de Ciências Sociais Aplicadas, 
Revista Ambiente Contábil - ISSN 2176-9036 - UFRN - Natal-RN. v. 8. n. 1, p. 258 - 274, jan./jun. 2016.

Curso de Mestrado em Administração. Endereço: Rua Imaculada Conceição, 1155 - Bloco

Acadêmico Prado Velho - CEP 80215901 - Curitiba, PR - Brasil - Telefone: (41) 32711476

Fax: (41) 32711638

Email: carlos.quandt@pucpr.br

[Artigo apresentado XXXVIII Encontro da ANPAD - EnANPAD 2014]

\title{
RESUMO
}

Este estudo visa identificar os indicadores mais significativos para o desenvolvimento de inteligência competitiva nas empresas da REDEPETRO-RN, que conta com 88 empresas associadas na cidade de Mossoró-RN. A amostragem não probabilística resultou em 59 respostas válidas. Trata-se de uma pesquisa aplicada do tipo survey, classificada como exploratória e descritiva quanto aos objetivos, com abordagem quantitativa e temporalidade cross-section. Os resultados indicaram que o envolvimento da alta direção nos sistemas de inteligência competitiva permite um monitoramento consistente do mercado e do comportamento da concorrência, que podem levar a modificações das estratégias deliberadas. A coleta de informações mercadológicas precisa de foco para apoiar eficazmente o processo decisório, e as informações devem ser compatíveis com os objetivos estratégicos por meio de instrumentos eficazes e fontes confiáveis.

Palavras-chave: Inteligência competitiva. Informações estratégicas. Processo decisório. Envolvimento da Alta Direção. REDEPETRO.

\begin{abstract}
The objective of this study is to identify the most significant contributors for the competitive intelligence development at REDEPETRO's companies in Rio Grande do Norte with 88 partner companies in the city of Mossoró-RN. The non probable sampling resulted in 59 valid responses. It involves a correlated research, such as a survey, classified as exploratory and descriptive of the goals on the central objectives abording quantitative and temporal crosssection. The results indicated that the involvement of high direction in the competitive intelligence systems allows consistent market monitoring and competitive performance that can lead modifications to deliberate strategies.The gathering is market information needs to be able to focus on effective supporting decision making processes and the information must be compatible with the strategic goals with the assistance of effective tools and reliable sources.

Keywords: Competitive intelligence. Strategic information. Decision making process. High direction involvement or senior management. REDEPETRO
\end{abstract}

\section{RESUMEN}

Este estudio busca identificar los indicadores más significativos para el desenvolvimiento de inteligencia competitiva en las empresas de la REDEPETRO-RN, con 88 empresas asociadas en la ciudad de Mossoró-RN. La muestra no probabilística resultó en 59 respuestas válidas. Se trata de una investigación tipo survey, clasificada como exploradora y descriptiva en relación a los objetivos, con abordaje cuantitativo e temporalidad cross-section. Los resultados indicaron que el envolvimiento de la alta dirección en los sistemas de inteligencia competitiva permite un monitoreo consistente del mercado y comportamiento de la competencia que pueden llevar a modificaciones de las estrategias deliberadas. La recolección de informaciones mercadológicas necesita enfoque para apoyar eficazmente el proceso decisivo, y las informaciones deben ser compatibles con los objetivos estratégicos por medio de instrumentos eficaces y fuentes confiables. 
Revista Ambiente Contábil - ISSN 2176-9036 - UFRN - Natal-RN. v. 8. n. 1, p. 258 - 274, jan./jun. 2016.

Palabras clave: Inteligencia Competitiva. Informaciones estratégicas. Proceso decisivo. Envolvimiento de la Alta Dirección. REDEPETRO

\section{INTRODUÇÃO}

As condições concorrenciais na economia contemporânea são fortemente influenciadas pelos rápidos avanços nas tecnologias de informação e comunicação (TICs), pela redução de barreiras comerciais e pela consequente abertura de mercados regionais e nacionais a novos concorrentes. Essas mudanças afetam, em maior ou menor grau, todas as organizações empresariais, independentemente de seu porte, setor ou localização. A capacidade de detectarem as ameaças do ambiente externo e de se antecipar a elas, bem como de aproveitar as oportunidades mercadológicas se configura como uma exigência básica para enfrentar o movimento das forças competitivas (BLENKHORN, 2005).

Nesse contexto, a informação mercadológica assume um papel fundamental na condução dos negócios. Considerando o conhecimento como um recurso estratégico na sociedade pós-industrial, a conquista de vantagens competitivas sustentáveis pode ser obtida por empresas que conseguem transformar informações de mercado em conhecimento organizacional para a tomada de decisões estratégicas (YAP et al., 2012).

A estruturação de sistemas de inteligência competitiva ancorados na tecnologia da informação e em processos organizacionais bem definidos de gestão do conhecimento frequentemente integra a estratégia adotada para captura, seleção, armazenagem e aplicação da informação. A utilidade de tais sistemas depende do estabelecimento de parâmetros e indicadores empresariais compatíveis com a realidade mercadológica em que a organização está inserida, com o objetivo de criar sistemas de inteligência competitiva adequados às necessidades de informação dos responsáveis pelas decisões estratégicas (WALTERS et al., 2005).

Compreende-se que um sistema de inteligência competitiva captura e processa informações de mercado e é necessário a empresas de qualquer setor, principalmente àquelas que estão inseridas em setores dinâmicos e caracterizados por alta concorrência. Verifica-se que a indústria de petróleo e gás triplicou a sua participação no valor da transformação industrial brasileira em uma década (SANT'ANNA, 2010), além de ampliar as exportações e reduzir as importações do setor. Com a expansão da cadeia produtiva do petróleo, empresas que atuam no setor buscam estratégias de fortalecimento da competitividade. Nesse contexto, foi criada a REDEPETRO BRASIL, que é uma organização para a integração das empresas fornecedoras de bens e serviços da cadeia de petróleo e gás natural por meio de articulação e convênios com governos, instituições acadêmicas e o Serviço Brasileiro de Apoio às Micro e Pequenas Empresas (SEBRAE). A promoção de eventos, a cooperação e ações diversas visam ao fortalecimento empresarial e ao aumento da competitividade das organizações que compõem a cadeia produtiva de petróleo e gás.

A REDEPETRO está presente em diversos estados da federação, como: Alagoas, Bahia, Ceará, Amazonas, Pernambuco, Espírito Santo, Minas Gerais, Paraná, Sergipe, Rio Grande do Sul, etc. No Rio Grande do Norte, a REDEPETRO está concentrada principalmente na região do município de Mossoró, área geográfica caracterizada pela exploração de petróleo e gás natural, que tem um papel significativo na economia local.

A presente pesquisa foi motivada principalmente pela inexistência de estudos empíricos sobre o papel da inteligência competitiva no processo de gestão de informações mercadológicas por essas empresas, no contexto de seus esforços para o fortalecimento da gestão empresarial e aumento da competitividade. O estudo justifica-se ainda pela importância estratégica dessa indústria para a economia do estado do Rio Grande do Norte e principalmente para o município de Mossoró, bem como pela relevância da REDEPETRO em 
Revista Ambiente Contábil - ISSN 2176-9036 - UFRN - Natal-RN. v. 8. n. 1, p. 258 - 274, jan./jun. 2016.

congregar empresas distintas que necessitam atuar competitivamente em uma cadeia produtiva dinâmica. Nessa perspectiva, esta pesquisa busca elucidar o seguinte questionamento: Quais os indicadores mais significativos para o desenvolvimento de inteligência competitiva, das empresas cadastradas na REDEPETRO que atuam no município de Mossoró?

\section{REVISÃO DA LITERATURA}

\subsection{INTELIGÊNCIA COMPETITIVA}

A inteligência competitiva tem raízes nas atividades de inteligência associadas à estratégia militar e à segurança nacional, cujo foco está nas potenciais ameaças de forças hostis (PRESCOTT, 1999). A partir da década de 1980, a inteligência competitiva ganhou notoriedade no campo da administração, particularmente por sua associação com a visão de estratégia competitiva proposta por Michael Porter, que argumenta que "o desenvolvimento de uma estratégia competitiva é, em essência, o desenvolvimento de uma fórmula ampla para o modo que uma empresa irá competir" (PORTER, 1986, p. 15). O conceito de inteligência competitiva passou a ser aplicado no mundo dos negócios como ferramenta de apoio gerencial à tomada de decisão estratégica, segundo Miller (2002, p. 35), "no entendimento de que os gerentes precisam estar sempre bem informados sobre questões fundamentais de negócios de maneira formal e sistêmica".

No Brasil, a partir de meados da década de 1990, a inteligência competitiva ganhou um auxílio significativo, para sua difusão como técnica, dos programas das universidades em seus cursos de pós-graduação e da criação da Associação Brasileira dos Analistas de Inteligência Competitiva (ABRAIC) em 2000, que surgiu no campo empresarial, com o objetivo de promover as práticas de inteligência competitiva entre as empresas brasileiras (Mendes et al, 2010). No âmbito internacional, a Strategic and Competitive Intelligence Professionals (SCIP) é uma organização global sem fins lucrativos, criada em 1986, que reúne profissionais de estratégia e inteligência competitiva. Presente em mais de 70 países, a SCIP tem cerca de 3.000 membros.

A inteligência competitiva, segundo Miller (2002, p. 25), "trata da análise das informações sobre o mercado e da geração de recomendações para os que decidem dentro das empresas". Para Tarapanoff (2001, p. 45), a IC pode ser considerada uma metodologia que permite o monitoramento informacional do ambiente externo da organização e deve tornar-se uma prática integrante do processo de gestão estratégica das organizações, uma vez que ela previne eventos que possam afetar o desempenho e o sucesso organizacional.

Tyson (1998) utiliza o conceito de inteligência competitiva a partir de uma perspectiva mais estratégica, como uma disciplina capaz de integrar três importantes atividades empresariais: planejamento estratégico, marketing e gestão da informação. Para ele, os esforços de inteligência objetivam o monitoramento constante e sistemático do ambiente externo de maneira rápida e precisa. Prescott e Miller (2002), por sua vez, conceituam inteligência competitiva como o processo de coletar, analisar e aplicar, legal e eticamente, informações sobre as condições competitivas dos concorrentes, como capacidades (forças e fraquezas) e intenções, além das forças macroambientais ou gerais que possam ter alguma importância para a tomada de decisão estratégica e o planejamento competitivo.

O papel da inteligência competitiva nas empresas pode ser classificado em cinco categorias gerais: 1) apoio à tomada de decisão estratégica; 2) identificação e antecipação de ameaças ao negócio; 3 ) apoio ao planejamento estratégico e implementação de atividades de marketing, gestão da tecnologia de informação e pesquisa e desenvolvimento; 4) apoio ao 
Revista Ambiente Contábil - ISSN 2176-9036 - UFRN - Natal-RN. v. 8. n. 1, p. 258 - 274, jan./jun. 2016.

monitoramento da concorrência e 5) apoio a atividades de benchmarking (GELB et al. 1991; VEDDER; GUYNES 2000; YAP et al., 2012)

$\mathrm{Na}$ visão de Correia (2006), a inteligência competitiva é o processo direcionado e planejado pelas necessidades de informação da organização, ilustrado na Figura 1.

\section{Figura 1 - Processo de inteligência competitiva}

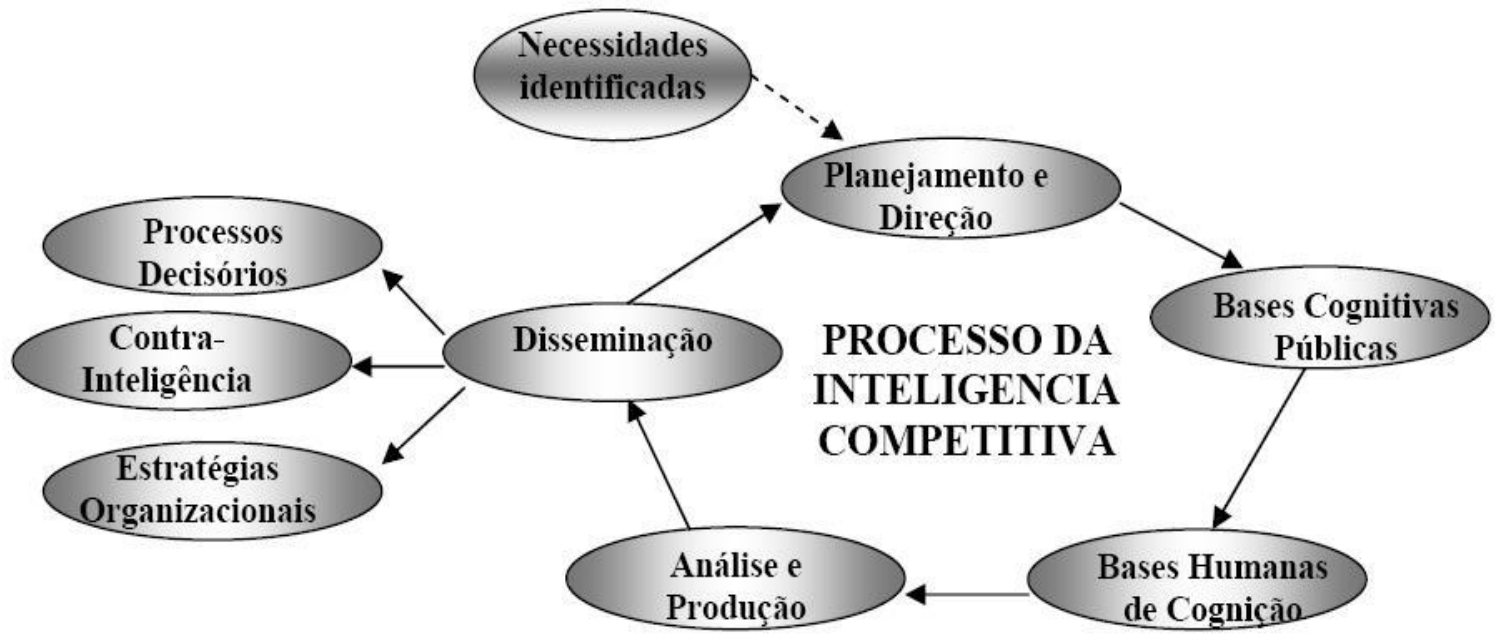

Fonte: Adaptada de Correia (2006, p. 53).

As necessidades de informação são normalmente definidas pelos objetivos estratégicos e seus detalhamentos, que determinam tempo, recursos e resultados a serem alcançados. As informações são buscadas em duas fontes: as bases cognitivas públicas e as pessoais. Tais informações são, então, selecionadas e produzidas (compactadas ou interpretadas) de acordo com as necessidades organizacionais. Finalmente, as informações assim produzidas são disseminadas às bases de conhecimento demandantes na organização.

\subsection{INTELIGÊNCIA COMPETITIVA E GESTÃO DO CONHECIMENTO}

A capacidade de adaptação a mudanças no mercado e nas ações dos concorrentes é um componente essencial para a obtenção de vantagens competitivas sustentáveis. Tecnologia, sistemas de gestão da qualidade, logística, preços competitivos e até mesmo o porte da empresa não mais se configuram, nos dias atuais, como vantagem competitiva duradoura. Segundo Canongia et al. (2001), a aceleração dos avanços científicos e tecnológicos e o ritmo crescente do desenvolvimento econômico a partir da segunda metade do século XX criam novos desafios em mercados globalizados e crescentemente competitivos.

Nesse contexto, a capacidade de criar e aplicar conhecimento, bem como a obtenção de informações sobre mercados e concorrentes, permitem às organizações elaborar e executar ações em busca de inovações e competitividade. Informação, conhecimento, inovação e competitividade estão intimamente associados. Nonaka e Takeuchi (1998), ao analisarem a competitividade das empresas japonesas, concluíram que existe uma singularidade entre a busca de conhecimento nos meios interno e externo e que esse conhecimento externo acumulado é disseminado de maneira vasta dentro da organização, bem como acumulado e utilizado no desenvolvimento de novas tecnologias e produtos. Tarapanoff (2001) ressalta que, na sociedade da informação ou "pós-industrial", a hegemonia econômica e social migrou 
daqueles que detinham os meios de produção para aqueles que administram o conhecimento e podem planejar a inovação. A autora também afirma que uma das principais ferramentas para a gestão da informação é a inteligência competitiva, considerada por ela como uma "nova síntese teórica" no tratamento da informação para a tomada de decisão.

Sob essa perspectiva, a gestão do conhecimento e a inteligência competitiva se configuram como alternativas estratégicas na busca contínua das empresas por inovações e ganhos de competitividade. Segundo Wiig (1993), a Gestão do Conhecimento pode ser conceituada como a construção sistemática, explicita e intencional do conhecimento e sua aplicação para maximizar a eficiência e o retorno sobre ativos de conhecimento da organização. Beckman (1999) a define como a formalização de experiências, conhecimento e expertise, de forma que se tornem acessíveis para a organização e esta possa criar novas competências, alcançar desempenho superior, estimular a inovação e criar valor para seus clientes. Dalkir (2011) caracteriza a Gestão do Conhecimento como a coordenação deliberada e sistemática das pessoas, da tecnologia, dos processos e da estrutura de uma organização com o objetivo de adicionar valor, por meio do reuso do conhecimento e da inovação. Essa coordenação é alcançada por meio da criação, do compartilhamento e da aplicação do conhecimento, assim como pela absorção de lições aprendidas e melhores práticas na memória corporativa, com o objetivo de fomentar a aprendizagem organizacional.

A utilização de termos como coordenação, compartilhamento e aprendizagem refletem a percepção de que não é possível fazer a gestão do conhecimento como se gerenciam outros ativos da organização. "O que se pode fazer é gerenciar o ambiente no qual o conhecimento pode ser criado, descoberto, capturado, compartilhado, destilado, validado, transferido, adotado, adaptado e aplicado" (COLLISON; PARCELL, 2005, p.24). Esse argumento se deve à necessidade de se estabelecer uma distinção entre informação e conhecimento. Este é a combinação da informação com experiência, contexto, interpretação e reflexão, que propiciam uma estrutura para avaliar e absorver novas experiências e informações. Portanto, o conhecimento, diferentemente da informação, envolve necessariamente elementos pessoais, como valores e aspectos tácitos do saber. Porém, uma parte desse conhecimento pode ser explicitado e absorvido pelas organizações, tanto em repositórios quanto em rotinas, processos, práticas e normas (DAVENPORT; PRUSAK, 1998). Nesse processo, o conhecimento tácito é convertido em conhecimento explícito.

Segundo Carvalho (2006), em um sistema de inteligência competitiva, a informação externa é sintetizada e interpretada pela empresa. A inteligência competitiva está voltada para a obtenção de informações de mercado que possibilitem à organização se antecipar e compreender cenários atuais e futuros. Para Fuld (2007), a linguagem secreta da inteligência competitiva ancora-se em dois pilares: o primeiro é encontrar a correta informação a respeito da competitividade; o segundo e mais significativo diz respeito à capacidade da organização de enxergar disrupções de mercado e interpretar corretamente os eventos.

Assim, a inteligência competitiva pode ser vista como um subconjunto da gestão do conhecimento. Enquanto esta última trata do conhecimento organizacional como um todo, incluindo os seus componentes tácitos e explícitos, a inteligência competitiva concentra-se no monitoramento do ambiente externo. Portanto, as duas abordagens são complementares do ponto de vista estratégico, pois procuram sistematizar a captação, a análise e a aplicação de informações e a sua conversão em conhecimento contextualizado, com o objetivo de apoiar a tomada de decisões, gerar inovações, antecipar ameaças, explorar oportunidades, e, de maneira mais ampla, aumentar a competitividade da organização (YAP et al., 2012).

Segundo Sabherwall e Fernandez (2010), para existir um sistema de inteligência competitiva que possa contribuir para a tomada de decisões estratégicas, a empresa precisa direcionar o fluxo interno das informações, bem como compreender como a informação é utilizada em toda a organização. Um sistema de inteligência competitiva busca monitorar os 
Revista Ambiente Contábil - ISSN 2176-9036 - UFRN - Natal-RN. v. 8. n. 1, p. 258 - 274, jan./jun. 2016.

movimentos da concorrência e outras forças, bem como as mudanças mercadológicas e tecnológicas que se caracterizem como ameaça ao negócio. Contudo, isso não significa que a inteligência competitiva também não busque oportunidades de mercado. $\mathrm{Na}$ realidade, inteligência competitiva é um instrumento de análise do ambiente externo em busca de se antecipar às "intempéries" do mercado, permitindo à empresa agir estrategicamente. Segundo Janissek-Muniz, Lesca e Freitas (2006), o conhecimento é uma necessidade básica para uma decisão estratégica. A empresa deve possuir o maior número de informações sobre o ambiente competitivo, conhecer e monitorar as ações dos concorrentes para poder agir com rapidez. A Figura 2 ilustra os níveis de conhecimento do ambiente.

\section{Figura 2 - Nível de conhecimento do ambiente}

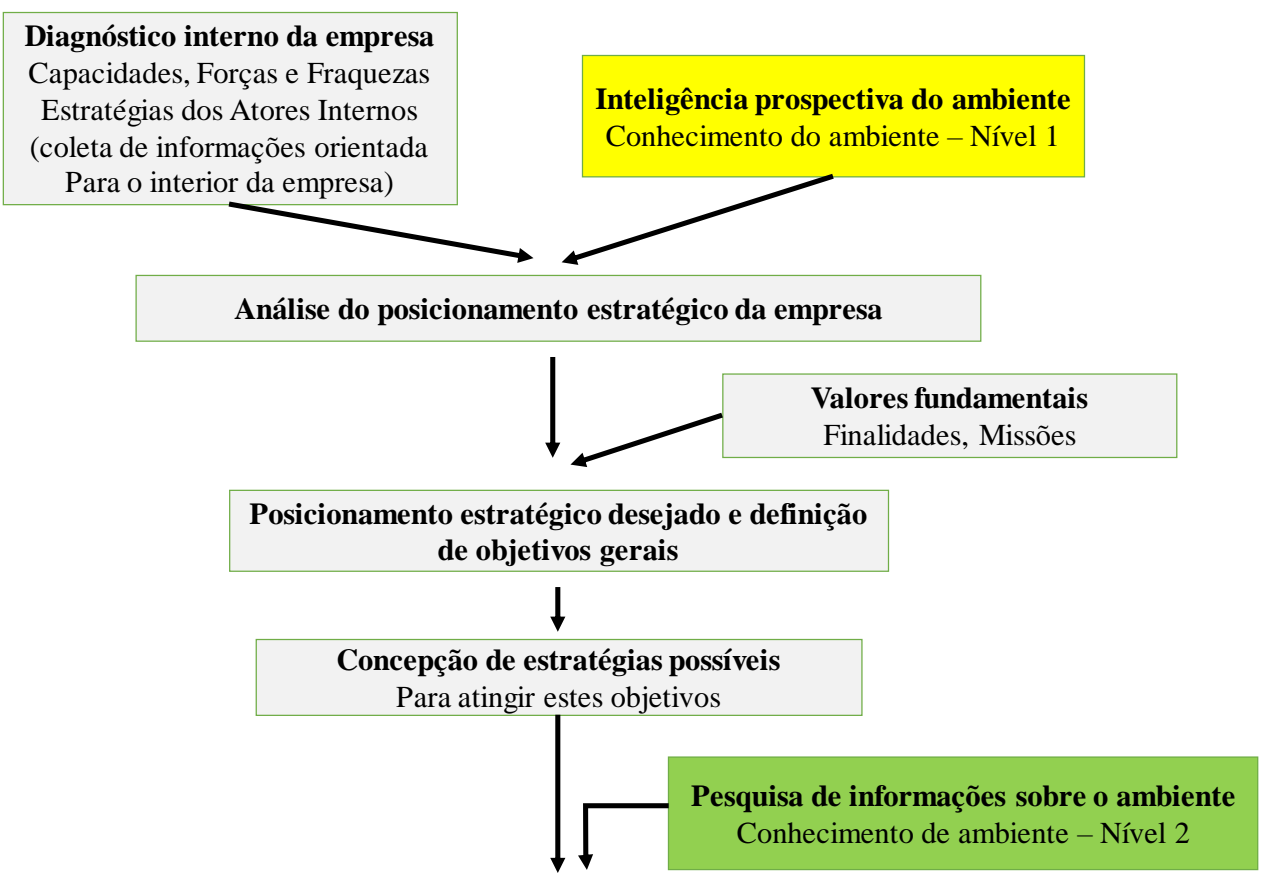

Fonte: Inteligência para Decisão Estratégica. Janissek-Muniz, Lesca e Freitas (2006).

Os sistemas de inteligência competitiva necessitam de métodos e estrutura adequados para a captura de informações, processamento e transformação em estratégias empresariais, de forma deliberada ou emergente. Os sistemas não se limitam ao monitoramento, mas também à construção de cenários com o objetivo de vislumbrar tendências futuras. Fuld (2007) lança uma reflexão nesse sentido quando questiona como as empresas se preparam para os concorrentes que ainda não existem. Ele ressalta que a construção de uma visão nítida de cenários competitivos distantes é feita com disciplina e estrutura. Percebe-se que os sistemas de inteligência competitiva complementam a gestão do conhecimento organizacional no sentido de captar e processar informações externas e transformá-las em conhecimento para a tomada de decisões, bem como de identificar tendências mercadológicas que propiciam um melhor posicionamento estratégico em relação à concorrência, conseguindo, assim, ganhos em competitividade empresarial.

\section{FUNDAMENTAÇÃO E HIPÓTESES DA PESQUISA}

Dentre a escassa literatura que aborda, de forma empírica e simultânea, os temas Inteligência Competitiva, Envolvimento da Alta Direção e Desempenho Organizacional, destaca-se o estudo de Panizzon (2010), por se alinhar diretamente com os objetivos desta 
Revista Ambiente Contábil - ISSN 2176-9036 - UFRN - Natal-RN. v. 8. n. 1, p. 258 - 274, jan./jun. 2016.

pesquisa. Para analisar a relação entre o envolvimento da alta direção e as dimensões de contexto e de processo de inteligência competitiva nas empresas de médio e grande porte da REDEPETRO no município de Mossoró-RN, o presente estudo teve como base, para o seu instrumento e suas hipóteses de pesquisa, a adaptação daqueles indicadores utilizados por Panizzon (2010). Desse modo, para identificar os indicadores mais significativos para o desenvolvimento de inteligência competitiva segundo o entendimento da alta direção, são propostas as seguintes hipóteses:

H1: Quanto maior o envolvimento da alta direção com a inteligência competitiva, maior é o desempenho da organização;

H2: Quanto maior a avaliação e a integração do processo decisório, maior é o envolvimento da alta direção;

H3: A coleta de informações de inteligência competitiva afeta positivamente o desempenho da organização;

H4: Quanto maior a coleta de informações de inteligência competitiva, maior é o envolvimento da alta direção.

Com base nessas hipóteses, o objetivo da pesquisa é auxiliar as empresas com informações, em função da contrapartida de disponibilização dos dados. Dessa maneira, em relação ao feedback dos resultados, pretende-se disponibilizar esta pesquisa às empresas e contribuir com estas para seu desenvolvimento com a inteligência competitiva em relação às onze dimensões (alerta e cultura, envolvimento da alta direção, infraestrutura, envolvimento dos funcionários, planejamento e foco, coleta, análise, disseminação, integração com o processo decisório, avaliação e desempenho da organização).

\section{METODOLOGIA}

A pesquisa se desenvolveu com as empresas cadastradas na REDEPETRO que atuam no município de Mossoró-RN, por meio de formulários cuidadosamente estruturados e aplicados à alta direção dessas empresas. Tem natureza aplicada, é do tipo survey, que, segundo Freitas et al. (2000), é o método indicado quando o interesse é produzir descrições quantitativas de uma população, fazendo uso de um instrumento predefinido. Quanto aos objetivos, é exploratória e descritiva, possui abordagem quantitativa e, em relação ao número de momentos, é cross-section. A REDEPETRO-RN conta com um total de 131 empresas associadas, das quais foi realizado um recorte para a cidade de Mossoró-RN, reduzindo o universo para um total de 88 empresas. A amostragem escolhida foi, por conveniência, não probabilística e contou com a participação de 59 empresas por meio de seus dirigentes ou funcionários da alta direção. Das empresas participantes, três foram excluídas por constarem, nos seus respectivos questionários, respostas em branco, ou por apresentarem caráter tendencioso, tais como a maioria das questões com respostas em uma mesma coluna. Foram consideradas válidas $n=56$, equivalente a $63,6 \%$ do total de empresas.

O instrumento de pesquisa utilizado foi adaptado de Panizzon (2010), fazendo-se uma adequação da quantidade de variáveis manifestas com o número de empresas participantes. Panizzon (2010) realiza um criterioso processo de construção e validação desse instrumento, tendo como base os questionários utilizados por Calof (2001), Saayman et al. (2008) e Viviers et al. (2005). Desse modo, partiu-se de um instrumento com 41 questões, distribuídas em 11 dimensões, para conhecer quais os indicadores mais significativos para o desenvolvimento de inteligência competitiva, segundo o entendimento da alta direção das empresas pesquisadas (Quadro 1). Dentre as principais diferenças entre o instrumento de pesquisa utilizado neste estudo e aquele proposto por Panizzon (2010), destacam-se a redução de 58 para 41 variáveis 
manifestas, a supressão de oito itens do questionário relacionados ao perfil da empresa respondente, além da adaptação de alguns outros itens. A necessidade de um questionário já validado, que contemple os objetivos deste estudo e a redução de itens do instrumento de pesquisa utilizado, deve-se ao baixo número de observações da amostra, $\mathrm{n}=56$ (correspondente a 63,6\% do universo de estudo). Não obstante, as metodologias utilizadas nos dois estudos são diferentes, fortalecendo a consistência na convergência de seus resultados.

O questionário utilizado na pesquisa mensura a percepção dos respondentes mediante três escalas de Likert, que variam de acordo com as dimensões que estão contemplando. $\mathrm{Na}$ primeira parte, trabalha as dimensões D1, D2, D3 e D4, nas quais cada quesito varia de 1 "discordo totalmente" para 5 "concordo totalmente". A segunda parte contém as dimensões D5, D6, D7, D8, D9 e D10, variando de 1 "nunca" para 5 "sempre". A terceira e última parte do instrumento de pesquisa compara o desempenho da empresa com os seus principais concorrentes nos últimos três anos e está representada pela dimensão D11 e sua escala varia de 1 "muito pior que os concorrentes" para 5 "muito melhor que os concorrentes".

Utilizou-se a técnica da Análise Fatorial Exploratória, que, segundo Marôco (2011), é uma técnica que tem por objetivo descobrir e analisar a estrutura de um conjunto de variáveis inter-relacionadas, de modo a construir uma escala de medida para fatores (intrínsecos) que, de alguma forma, controlam as variáveis originais.

Com o uso da Summated Scales, foram obtidos os construtos, que permitiram testar as hipóteses sobre as correlações. O presente estudo fez uso do software SPSS (Statistical Package for Social Science), versão 21.0.

\section{ANÁLISE DOS RESULTADOS}

A REDEPETRO está presente em todo o Brasil e, na cidade de Mossoró-RN, é composta de empresas que atuam no setor de petróleo e gás, com 62,5\% destas atuantes no setor de serviço. As empresas participantes da pesquisa apresentam um quadro de funcionários variando de 02 a 587. Das empresas que compõem a rede, 44,6\% praticam muita parceria/cooperação/aliança de qualquer tipo com outras empresas, seja no mercado interno, seja no externo, de acordo com a entrevista realizada com a alta direção dessas empresas.

O Quadro 1 apresenta as dimensões contempladas no instrumento de pesquisa com seus respectivos coeficientes alfa de Cronbach, que mede a consistência interna dos indicadores, baseada na correlação média entre as variáveis. É considerado por alguns autores como o método mais comum para análise da confiabilidade dos dados, tendo a ideia de que os indicadores principais devem medir o mesmo construto e serem intercorrelacionados (LEE; HOOLEY, 2005). Os valores do alfa de Cronbach variam de 0 a 1 , sendo os valores de 0,60 a 0,70 os limites inferiores de aceitabilidade, de acordo com Hair Jr. et al. (2009). A dimensão D5 - Planejamento e Foco, cujo alfa é de 0,547, necessita de uma maior cautela em sua análise, podendo haver sido subestimada por não fazer suposições relativas à homogeneidade das variâncias-covariâncias inter-itens (Marôco e Garcia-Marques, 2006), ou pelo simples fato de essa dimensão ter apenas três variáveis manifestas. No entanto, faz-se necessária no instrumento de pesquisa, devido sua importância para o tema abordado, considerando também o fato de esta dimensão apresenta alfa igual a 0,757 no estudo de Panizzon (2010), podendo haver sido influenciado pelo número de observações deste estudo $(\mathrm{n}=56)$. As demais dimensões atendem a esse critério, o que mostra a adequação do instrumento utilizado. 
Revista Ambiente Contábil - ISSN 2176-9036 - UFRN - Natal-RN. v. 8. n. 1, p. 258 - 274, jan./jun. 2016.

\begin{tabular}{|c|c|}
\hline \multicolumn{2}{|c|}{ D1 - Alerta e cultura } \\
\hline Ind.1 & $\begin{array}{l}\text { Nossa empresa é ágil para detectar eventos relevantes no mercado e mudanças fundamentais em } \\
\text { nosso ramo de atividade em curtos intervalos de tempo (por exemplo, preferência dos clientes, } \\
\text { concorrência, tecnologia, legislação). }\end{array}$ \\
\hline Ind.2 & $\begin{array}{l}\text { Nossa empresa incentiva o compartilhamento da informação, e os funcionários entendem que essa } \\
\text { prática é importante para o sucesso da empresa. }\end{array}$ \\
\hline Ind.3 & $\begin{array}{l}\text { Nossa empresa reconhece a inteligência competitiva (coleta, análise e disseminação sistemática } \\
\text { de informações para a tomada de decisão) como uma atividade necessária para a competividade } \\
\text { do negócio. }\end{array}$ \\
\hline \multicolumn{2}{|c|}{ D2 - Envolvimento da alta direção } \\
\hline Ind.4 & $\begin{array}{l}\text { A alta direção compreende o que é inteligência competitiva (atividade de coleta, análise e } \\
\text { disseminação de informações para a tomada de decisão). }\end{array}$ \\
\hline Ind.5 & $\begin{array}{l}\text { A alta direção usa os resultados de inteligência competitiva no seu planejamento estratégico na } \\
\text { tomada de decisão. }\end{array}$ \\
\hline Ind.6 & A alta direção apresenta uma atuação efetiva com relação às atividades de inteligência competitiva. \\
\hline \multicolumn{2}{|c|}{ D3 - Infraestrutura } \\
\hline Ind.7 & $\begin{array}{l}\text { Em nossa empresa, dispomos de um sistema organizado de fontes de informação que facilita o } \\
\text { armazenamento e a consulta de informações competitivas de que os funcionários possam necessitar } \\
\text { em seu trabalho. }\end{array}$ \\
\hline Ind.8 & $\begin{array}{l}\text { Em nossa empresa, temos canais convenientes para os funcionários reportarem suas observações e } \\
\text { informações. }\end{array}$ \\
\hline Ind.9 & $\begin{array}{l}\text { As pessoas responsáveis pela busca das informações comunicam-se diretamente com a alta direção } \\
\text { da empresa. }\end{array}$ \\
\hline \multicolumn{2}{|c|}{ D4 - Envolvimento dos funcionários } \\
\hline Ind.10 & A maioria dos funcionários compreende o que é inteligência competitiva. \\
\hline Ind.11 & $\begin{array}{l}\text { Nossa empresa tem incentivos e reconhecimentos para encorajar os funcionários a reportar e } \\
\text { compartilhar observações e informações de mercado. }\end{array}$ \\
\hline Ind.12 & Em nossa empresa, as necessidades de informação são comunicadas aos nossos funcionários. \\
\hline Ind.13 & $\begin{array}{l}\text { Nossos funcionários reportam informação sobre nossos competidores de mercado para os } \\
\text { tomadores de decisão. }\end{array}$ \\
\hline \multicolumn{2}{|c|}{ D5 - Planejamento e foco } \\
\hline Ind.14 & $\begin{array}{l}\text { Em nossa empresa, as necessidades de informações estratégicas são identificadas em reuniões com } \\
\text { a alta direção. }\end{array}$ \\
\hline Ind.15 & $\begin{array}{l}\text { Nossa empresa treina/prepara os nossos funcionários antes de eles participarem de eventos, } \\
\text { convenções, feiras sobre quais informações eles deverão procurar. }\end{array}$ \\
\hline Ind.16 & $\begin{array}{l}\text { Em nossa empresa, os projetos/atividades de busca de informações são gerados em função da } \\
\text { demanda de clientes internos. }\end{array}$ \\
\hline \multicolumn{2}{|c|}{ D6 - Coleta } \\
\hline Ind.17 & $\begin{array}{l}\text { Em nossa empresa, coletamos informação estratégica por meio de uma rede de relacionamentos } \\
\text { (parceiros, clientes, fornecedores...) que construímos para esse fim. }\end{array}$ \\
\hline Ind.18 & $\begin{array}{l}\text { Nossa empresa mantém uma postura de reciprocidade na troca de informações com redes de } \\
\text { relacionamento. }\end{array}$ \\
\hline Ind.19 & $\begin{array}{l}\text { Em nossa empresa, utilizamos ferramentas (softwares) para a coleta sistemática de informações na } \\
\text { internet, de modo a monitorar nossos ambientes de interesse. }\end{array}$ \\
\hline Ind.20 & Nossa empresa coleta o máximo de informações possíveis sobre os competidores. \\
\hline \multicolumn{2}{|c|}{ D7 - Análise } \\
\hline Ind.21 & $\begin{array}{l}\text { Em nossa empresa, as informações que obtemos passam pela análise de pessoas especialistas no } \\
\text { assunto tratado antes de serem utilizadas no processo decisório. }\end{array}$ \\
\hline Ind.22 & $\begin{array}{l}\text { As avaliações de informações da nossa empresa são pautadas por recomendações, ou informam } \\
\text { qual o significado/impacto daquela informação para a organização. }\end{array}$ \\
\hline Ind.23 & $\begin{array}{l}\text { Nossa empresa usa modelos básicos de análise (matriz swot, forças de Porter, cadeia de valor... ou } \\
\text { modelos desenvolvidos pela empresa). }\end{array}$ \\
\hline \multicolumn{2}{|c|}{ D8 - Disseminação } \\
\hline In & $\begin{array}{l}\text { Em nossa empresa, divulgamos as informações estratégicas para os colaboradores dos setores aos } \\
\text { quais essas informações dizem respeito. }\end{array}$ \\
\hline Ind.25 & $\begin{array}{l}\text { Em nossa empresa, as informações estratégicas são apresentadas numa variedade de maneiras } \\
\text { (briefings, relatórios, perfis de competidores, newletters). }\end{array}$ \\
\hline
\end{tabular}


Revista Ambiente Contábil - ISSN 2176-9036 - UFRN - Natal-RN. v. 8. n. 1, p. 258 - 274, jan./jun. 2016.

\begin{tabular}{|l|l|}
\hline Ind.26 & $\begin{array}{l}\text { Informações analisadas sobre o mercado (clientes, concorrência, legislação...) são sempre } \\
\text { amplamente disseminadas nesta empresa. }\end{array}$ \\
\hline D9 - Integração com o processo decisório & alfa = 0,860 \\
\hline Ind.27 & $\begin{array}{l}\text { Em nossa empresa, os gestores costumam decidir orientando-se pelas análises geradas pelas } \\
\text { atividades de inteligência competitiva. }\end{array}$ \\
\hline Ind.28 & $\begin{array}{l}\text { Em nossa empresa, os relatórios de inteligência competitiva servem como insumo para os } \\
\text { processos de planejamento (estratégico, marketing, operações...). }\end{array}$ \\
\hline Ind.29 & $\begin{array}{l}\text { Em nossa empresa, as informações obtidas em nosso ambiente de negócios são utlizadas para } \\
\text { fomentar inovação. }\end{array}$ \\
\hline Ind.30 & $\begin{array}{l}\text { Em nossa empresa, as decisões são tomadas com base em análises, dados e fatos, e não pelo } \\
\text { "achismo". }\end{array}$ \\
\hline D10 - Avaliação \\
\hline Ind.31 & $\begin{array}{l}\text { Em nossa empresa, há uma avaliação da qualidade de nossos produtos de inteligência competitiva } \\
\text { (briefings, relatórios, perfis de competidores, newletters). }\end{array}$ \\
\hline Ind.32 & $\begin{array}{l}\text { Existem questionamentos críticos e periódicos sobre o desempenho das atividades de inteligência } \\
\text { competitiva e das informações geradas. }\end{array}$ \\
\hline Ind.33 & $\begin{array}{l}\text { As avaliações sobre o desempenho das atividades de inteligência competitiva levam em conta } \\
\text { indicadores qualitativos e quantitativos. }\end{array}$ \\
\hline D11 - Desempenho da organização \\
\hline Ind.34 & Obtenção da satisfação dos clientes. \\
\hline Ind.35 & Manutenção dos clientes atuais. \\
\hline Ind.36 & Atração de novos clientes. \\
\hline Ind.37 & Participação de mercado. \\
\hline Ind.38 & Taxa de crescimento do faturamento. \\
\hline Ind.39 & Lucratividade do negócio (lucro operacional em relação ás vendas). \\
\hline Ind.40 & Produtos inovadores lançados no mercado. \\
\hline Ind.41 & \begin{tabular}{l} 
Desempenho geral da empresa. \\
\hline
\end{tabular} \\
\hline
\end{tabular}

Fonte: Elaborado pelos autores (2014).

\subsection{ANÁLISE FATORIAL EXPLORATÓRIA}

A análise fatorial exploratória tem como objetivo identificar a estrutura de um conjunto de variáveis e fornecer um processo para a redução de dados (HAIR Jr. et al., 2009). Dentre os diversos métodos de extração dos fatores, o mais utilizado, de acordo com Lemos, Frega e Souza (2010), é o da Análise dos Componentes Principais, que busca a combinação linear das variáveis, de modo que a variância extraída seja máxima. Desse modo, procedeu-se à utilização do método da Análise de Componente Principal, com o objetivo de reduzir o número de variáveis a um conjunto mais fácil de manipular (AAKER; KUMAR; DAY, 2004).

A adequação da Análise Fatorial Exploratória, para verificar se a matriz de correlação permite o seu uso, foi realizada pelo teste de esfericidade de Bartlett e a medida KaiserMeyer-Olkin (KMO). O teste de esfericidade de Bartlett rejeita a hipótese nula de que a matriz de correlação seja uma matriz identidade, ou seja, existe uma correlação significativa de pelo menos uma das variáveis. $\mathrm{O}$ indicador KMO acima de 0,7 e a alta significância obtida no teste de Bartlett ( $\mathrm{p}<0,001)$, apresentados na Tabela 1, indicam a utilização do método.

Tabela 1 -Teste de Bartlett e KMO

\begin{tabular}{llr}
\hline Kaiser-Meyer-Olkin Measure of Sampling Adequacy & & 0,781 \\
\hline \multirow{3}{*}{ Bartlett's Test of Sphericity } & Approx. Chi-square & 419,560 \\
\cline { 2 - 3 } & Df & 91 \\
\cline { 2 - 3 } & Sig. & 0,000 \\
\hline
\end{tabular}

Nota: Elaborada pelos autores (2014).

"O método de rotação utilizado é o Varimax, um método ortogonal que permite uma fácil interpretação dos dados" (MINGOTI, 2007, p. 121). A retenção dos fatores foi realizada 
Revista Ambiente Contábil - ISSN 2176-9036 - UFRN - Natal-RN. v. 8. n. 1, p. 258 - 274, jan./jun. 2016.

utilizando-se o Critério de Kaiser, valores próprios superiores a 1, sendo observado em conjunto com o critério do Scree plot (GRÁFICO 1), segundo recomendação de Marôco (2011), indicando a retenção de quatro fatores, capazes de explicar conjuntamente $74,89 \%$ da variância total.

\section{Figura 3 - Autovalores encontrados após extração dos componentes principais}

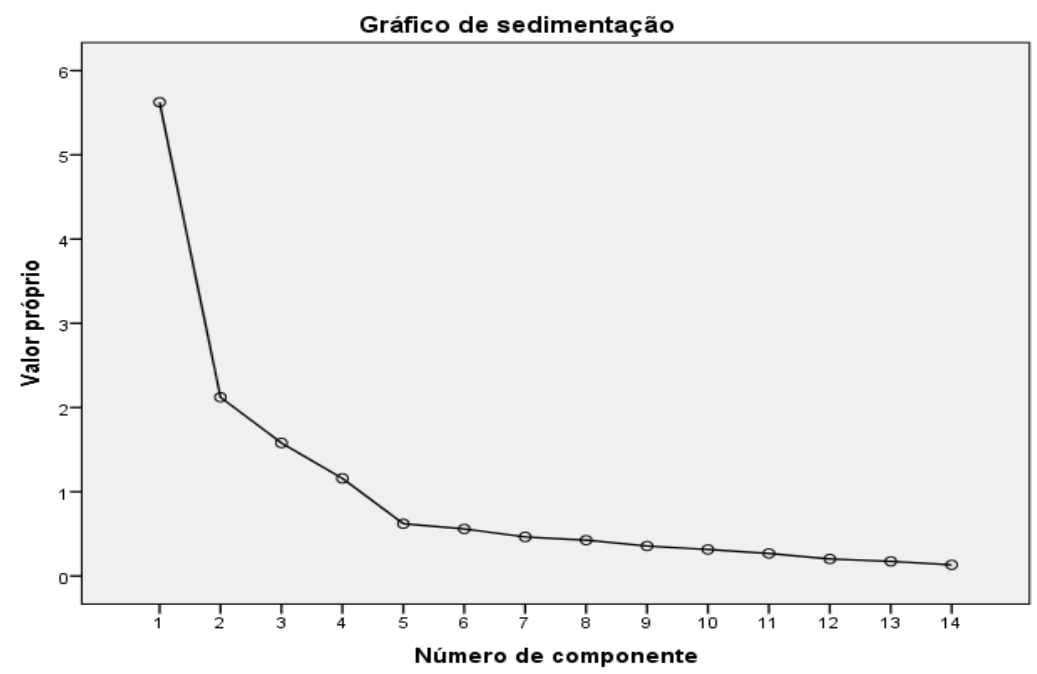

Fonte: Elaborado pelos autores (2014).

A Tabela 2 apresenta quatro fatores dotados de alta comunalidade $\left(h^{2}\right)$, que, de acordo com a opinião dos respondentes, são os que possuem maior nível de importância para o desenvolvimento de inteligência competitiva, segundo o entendimento da alta direção das empresas pesquisadas. A Tabela 2 apresenta ainda o valor próprio, o percentual da variância explicada e o alfa de Cronbach de cada uma das dimensões. "O valor próprio irá indicar que cada componente principal selecionado explique mais variância que cada uma das variáveis originais" (MARÔCO, 2011, p. 459). Desse modo, o fator que possui maior valor próprio possui um maior poder de explicação. Assim, o fator I, representado pelo "desempenho da organização", com valor próprio $=5,625$, é considerado como o de maior peso sobre o desenvolvimento de inteligência competitiva, tendo um peso bem mais acentuado que o fator II - "avaliação e integração do processo decisório", cujo valor próprio = 2,122 e assim sucessivamente. O percentual da variância total explicada irá denotar a contribuição de cada fator, destacando-se o valor de 17,216\% associado ao fator III - "envolvimento da alta direção", indicando a sua relevada importância para o desenvolvimento de inteligência competitiva no âmbito da empresa. O alfa de Cronbach fornece a confiabilidade de cada construto, segundo o qual se pode observar que os três primeiros fatores possuem valor maior que 0,8 , o que indica um bom nível de confiabilidade. O fator IV - "coleta de informações" possui alfa $=0,658$, que está dentro dos limites inferiores de aceitabilidade descrito por (HAIR et al., 2009, p. 126). 
Revista Ambiente Contábil - ISSN 2176-9036 - UFRN - Natal-RN. v. 8. n. 1, p. 258 - 274, jan./jun. 2016.

Tabela 2 - Solução fatorial com as cargas fatoriais, comunalidades, percentual de variância e alfa de Cronbach

\begin{tabular}{|c|c|c|c|c|c|}
\hline \multirow[t]{2}{*}{ Variável } & \multicolumn{4}{|c|}{ Fatores } & \multirow[t]{2}{*}{$h^{2}$} \\
\hline & I & II & III & IV & \\
\hline Ind.39 & $0,862 *$ & 0,081 & 0,145 & 0,024 & 0,840 \\
\hline Ind.38 & $\mathbf{0 , 8 4 9 *}$ & 0,018 & 0,141 & $-0,046$ & 0,759 \\
\hline Ind.41 & $0,766 *$ & 0,416 & 0,039 & 0,160 & 0,787 \\
\hline Ind.37 & $\mathbf{0 , 7 5 3 *}$ & 0,358 & 0,005 & 0,049 & 0,718 \\
\hline Ind.35 & $\mathbf{0 , 7 2 4 *}$ & 0,172 & 0,112 & 0,340 & 0,812 \\
\hline Ind.32 & 0,045 & $0,823 *$ & 0,234 & $-0,044$ & 0,710 \\
\hline Ind.29 & 0,256 & $0,787 *$ & 0,045 & 0,196 & 0,726 \\
\hline Ind.33 & 0,349 & $0,763 *$ & 0,102 & $-0,007$ & 0,736 \\
\hline Ind.28 & 0,136 & $0,752 *$ & 0,303 & 0,186 & 0,714 \\
\hline Ind.4 & 0,051 & 0,215 & $0,865^{*}$ & 0,206 & 0,682 \\
\hline Ind.6 & 0,142 & 0,058 & $0,844 *$ & 0,226 & 0,697 \\
\hline Ind.5 & 0,142 & 0,271 & $\mathbf{0 , 8 1 6 *}$ & $-0,008$ & 0,744 \\
\hline Ind.18 & 0,054 & $-0,073$ & 0,239 & $0,864 *$ & 0,772 \\
\hline Ind.17 & 0,154 & 0,303 & 0,109 & $0,768 *$ & 0,787 \\
\hline Valor Próprio (Eigenvalue) & 5,625 & 2,122 & 1,579 & 1,159 & \\
\hline \% Variância Total Explicada & 24,431 & 21,443 & 17,216 & 11,800 & \\
\hline Alfa de Cronbach & 0,884 & 0,852 & 0,847 & 0,658 & \\
\hline
\end{tabular}

Nota:*Cargas fatoriais significativas. $h^{2}$ - Comunalidade; Método de extração: Análise de componentes principais; Método de rotação: Varimax com normalização de Kaiser. Elaborada pelos autores (2014).

A Análise Fatorial Exploratória permitiu encontrar as dimensões: "desempenho da organização", "avaliação e integração do processo decisório", "envolvimento da alta direção" e "coleta de informações", representadas, respectivamente, pelos fatores I, II, III e IV. As dimensões representadas pelos fatores I, III e IV aparecem no instrumento de pesquisa, visto no Quadro 1 como D11, D2 e D6, respectivamente. Isso corrobora com o estudo feito por Panizzon (2010) no tocante à importância dessas dimensões para a generalização do estudo sobre o desenvolvimento de inteligência competitiva, bem como para o segmento de petróleo e gás, objeto deste estudo. Não obstante, a dimensão representada pelo fator II, que é a "avaliação e integração do processo decisório", desponta como uma nova dimensão para o estudo desse tema, aplicada a empresas que atuam no segmento de petróleo e gás, como sendo uma fusão das dimensões "integração para o processo decisório" e "avaliação", já consagrada por diversos autores em outros estudos (CALOF, 2001; PANIZZON, 2010; SAAYMAN et al., 2008; VIVIERS et al., 2005).

\subsection{TESTE DE HIPÓTESES SOBRE AS CORRELAÇÕES}

Para testar as hipóteses sobre as correlações, procedeu-se inicialmente à obtenção dos construtos obtidos na Análise Fatorial, por meio da Summated Scales, em que os fatores I, II, III e IV passam a ser representados pelos construtos $\mathrm{C} 1, \mathrm{C} 2, \mathrm{C} 3$ e $\mathrm{C} 4$, respectivamente.

As correlações dos construtos C1, C2, C3 e C4 são apresentadas na Tabela 3, na qual podemos constatar que todas as correlações foram significativas com $\mathrm{p}<0,05$. Considerando que a população pesquisada é de apenas 88 empresas e que foi obtido um total de 56 questionários considerados válidos, isso representa uma amostra de $63,6 \%$ da população, o que fornece um maior indicativo de segurança no estudo das correlações. As correlações $\mathrm{C} 1 \mathrm{e}$ C3, C1 e C4 possuem uma avaliação qualitativa considerada fraca em relação ao grau de correlação $(0<|r|<0,3)$; as demais correlações são ditas regulares $(0,3 \leq|r|<0,6)$, de acordo com Callegari-Jacques (2003). 
Revista Ambiente Contábil - ISSN 2176-9036 - UFRN - Natal-RN. v. 8. n. 1, p. 258 - 274, jan./jun. 2016.

Tabela 3 - Correlações

\begin{tabular}{llrrrr}
\hline & & C1 & C2 & C3 & \multicolumn{1}{c}{ C4 } \\
\hline \multirow{2}{*}{ C1 } & Correlação de Pearson & 1 & $0,481^{* *}$ & $0,297^{*}$ & $0,284^{*}$ \\
& Sig. (2 extremidades) & & 0,000 & 0,026 & 0,034 \\
\hline \multirow{2}{*}{ C2 } & Correlação de Pearson & $0,481^{* *}$ & 1 & $0,431^{* * *}$ & $0,302^{* *}$ \\
& Sig. (2 extremidades) & 0,000 & & 0,001 & 0,024 \\
\hline \multirow{2}{*}{ C3 } & Correlação de Pearson & $0,297^{*}$ & $0,431^{* *}$ & 1 & $0,374^{* *}$ \\
& Sig. (2 extremidades) & 0,026 & 0,001 & & 0,005 \\
\hline \multirow{2}{*}{ C4 } & Correlação de Pearson & $0,284^{*}$ & $0,302^{*}$ & $0,374^{* *}$ & 1 \\
& Sig. (2 extremidades) & 0,034 & 0,024 & 0,005 & \\
\hline
\end{tabular}

Nota: *A correlação é significativa no nível 0,05 (2 extremidades). **A correlação é significativa no nível 0,01 (2 extremidades). Elaborada pelos autores (2014).

A hipótese (H1) "quanto maior o envolvimento da alta direção com a inteligência competitiva, maior é o desempenho da organização" é verificada ao se observar a correlação entre $\mathrm{C} 1$ e C3, que é significativa com p<0,05. Entretanto, possui uma baixa carga de 0,297, o que reduziria a sua probabilidade de ocorrência se não houvesse uma amostra de $63,6 \%$ da população. A hipótese (H2) "quanto maior a avaliação e integração do processo decisório, maior é o envolvimento da alta direção" envolve C2 e C3, é altamente significativa com $\mathrm{p}<0,01$ e possui carga 0,431 , indicando uma maior probabilidade de ocorrência. A hipótese (H3) "a coleta de informações de inteligência competitiva afeta positivamente o desempenho da organização" correlaciona $\mathrm{C} 1$ e $\mathrm{C} 4$, é significativa com $\mathrm{p}<0,05$ e também possui uma carga considera baixa de 0,284, enquadrando-se no mesmo caso de (H1). Por fim, a hipótese (H4) "quanto maior a coleta de informações de inteligência competitiva, maior é o envolvimento da alta direção" relaciona os construtos $\mathrm{C} 3$ e C4, tem significância de p<0,01 e apresenta uma carga de 0,374 .

\section{CONSIDERAÇÕES FINAIS}

Os sistemas de inteligência competitiva necessitam do monitoramento e do apoio da alta direção para que sejam estruturados e efetivamente aplicados. O presente estudo contemplou 11 dimensões, representadas por 41 variáveis. O resultado da Análise Fatorial converge para quatro dimensões, no sentido de que evidenciam as variáveis mais significativas relacionadas ao uso da inteligência competitiva, à luz da alta direção das empresas que atuam no segmento de petróleo e gás associadas à REDEPETRO-RN.

As dimensões consideradas foram: "desempenho da organização", "avaliação e integração do processo decisório", "envolvimento da alta direção" e "coleta de informações".

A avaliação dos sistemas de inteligência competitiva e integração do processo decisório estão intimamente associadas. Uma vez que a arquitetura estratégica da organização tem como base as informações mercadológicas fornecidas pelos instrumentos de coleta e processamento do sistema, esses instrumentos precisam de revisão e avaliação constantes com o objetivo de compatibilizar a sua aplicação com os objetivos e contextos vigentes. $\mathrm{O}$ envolvimento da alta direção na estrutura dos sistemas de inteligência competitiva permite um monitoramento mais consistente das oscilações de mercado e do comportamento da concorrência, que podem levar a modificações das estratégias deliberadas. A coleta de informações mercadológicas precisa de foco e relevância para suprir o sistema de inteligência competitiva de uma base consistente para o processo decisório, assim como as informações coletadas precisam ser compatíveis com os objetivos propostos por meio de instrumentos eficazes e fontes confiáveis.

As quatro hipóteses propostas nesta pesquisa foram confirmadas, evidenciando que quanto maior for o envolvimento da alta direção com a inteligência competitiva, maior será o 
Revista Ambiente Contábil - ISSN 2176-9036 - UFRN - Natal-RN. v. 8. n. 1, p. 258 - 274, jan./jun. 2016.

desempenho da organização e também a avaliação e a integração do processo decisório. As hipóteses confirmaram ainda a importância sobre a coleta de informação de inteligência competitiva, indicando que esta afeta positivamente o desempenho da organização e depende do envolvimento da alta direção.

Dentre as limitações deste estudo destaca-se a não generalização de seus resultados, por não se tratar de um estudo probabilístico, limitando-se ao estudo das empresas associadas à REDEPETRO, situadas no polo petrolífero de Mossoró-RN. Outros estudos podem se aprofundar na pesquisa sobre o uso da inteligência competitiva pela alta direção, estendendose as demais localidades em que atuam empresas associadas a REDEPETRO. O referido artigo contribui na pesquisa teórica sobre estratégia na medida em que contemplou a associação entre inteligência competitiva e gestão do conhecimento como instrumentos de formulação estratégica das organizações, principalmente das estratégias deliberadas. Outros estudos podem se aprofundar nessa associação enfatizando outros aspectos da gestão estratégica, como por exemplo a influência dos sistemas de informações mercadológicas e gestão do conhecimento nas estratégias emergentes.

\section{REFERÊNCIAS}

BECKMAN, T. The current state of knowledge management. IN: Liebowitz, J. (ed) Knowledge Management Handbook. p.1.1-1.22. NY: CRC Press, 1999.

BLENKHORN, D. L. Competitive Intelligence and Global Business. Greenwood Publishing Group, 2005.

CALOF, J. Competitive intelligence and the small firm: requeriments and barriers. $46^{\text {th }}$ International Counicl on Small Business, 2001. Conference in Taipei, China, p. 17-20, June. Disponível em: 〈http://sbaer.uca.edu/research/icsb/2001/paper16.pdf>. Acesso em: 22 jun. 2013.

CANONGIA, C. et al. Convergência da inteligência competitividade com construção de visão do futuro: proposta metodológica de sistemas de informação estratégica (SIE). Revista de ciência da informação, Rio de Janeiro, v. 2 n. 3, junho.2001. Disponível em: < http://www.datagramazero.org.br/jun01/Art_02.htm>. Acesso em: 15 jun. 2013.

CARVALHO, H. Gestão do conhecimento e inteligência competitiva: sistemas complementares. 2006. Disponível em:

$<$ http://www.pg.utfpr.edu.br/ppgep/Ebook/ARTIGOS2005/Ebook\%202006_artigo\%2055.pdf $>$. Acesso em: 15 jun. 2013.

COLLISON, C,: Parcell, G. Learning to Fly: Practical Knowledge Management from Leading and Learning Organizations. Capstone, 2005.

CORREIA, C. C. . Getting competitive. Library journal, v. 131, n. 7, p. 52-54, 2006.

DALKIR, Kimitz. Knowledge management in theory and pratice. 2. ed. Burlington: Elsevier Butterworth-Heinemann, 2011.

DAVENPORT, T. H., PRUSAK, L. Working knowledge: How organizations manage what they know. Boston: Harvard Business School Press, 1998. 
Revista Ambiente Contábil - ISSN 2176-9036 - UFRN - Natal-RN. v. 8. n. 1, p. 258 - 274, jan./jun. 2016.

FULD, L. M. Inteligência competitiva: como se manter à frente dos movimentos da concorrência e do mercado. Rio de Janeiro: Elsevier, 2007.

GELB, B. D.; SAXTON, M. J.; ZINKHAN, G. M; Albers, N. D; Competitive intelligence: insights from executives. Business Horizons, V. 34, N.1, p. 43-47, 1991.

HAIR, JOSEPH F.; BLACK, WILLIAM C.; BABIN, BARRY J.; ANDERSON, ROLPH E.; TATHAM, RONALD L. Análise multivariada de dados. Trad. Adonai Schlup Sant'Anna. Rev. Maria Aparecida Gouvêa. 6. ed. Porto Alegre, Bookman, 2009.

JANISSEK, M. R.; LESCA, H.; FREITAS, H. Inteligência estratégica antecipativa e coletiva para tomada de decisão. Anais do 3rd CONTECSI - International Conference on Information Systems and Technology Management and 11th WCA - Word Continuous Auditing Conference. São Paulo, 2006.

MARÔCO, J. Análise estatística com o SPSS Statistics. 5. ed. Lisboa: ReportNumber, 2011.

MAROCO, J.; GARCIA-MARQUES, T.. Qual a fiabilidade do alfa de Cronbach? Questões antigas e soluções modernas? Laboratório Psicologia, 4, 65-90. 2006.

MENDES, A.; MARCIAL, E.; FERNANDES, F. Fundamentos da Inteligência Competitiva. Brasília: Thesaurus, 2010.

MILLER, Jerry P. O milênio da inteligência competitiva. Porto Alegre: Bookman, 2002.

MULLER, M. L.; JEGERS, M. Competitive intelligence: construct exploration, validation and equivalence. Aslib Proceedings: New Information Perspectives, v. 60, n. 4, p. 383411.2008.

NONAKA, I., TAKEUCHI, H. Criação do conhecimento na empresa: como as empresas japonesas geram a dinâmica da inovação. Rio de Janeiro: Campus, 1998.

PANIZZON, M. Envolvimento da alta direção e inteligência competitiva: uma análise sobre suas relações em organizações de médio e de grande porte da serra Gaúcha Brasil. Dissertação de Mestrado em Administração. Universidade de Caxias do Sul, Caxias do Sul, 2010.

PORTER, M. Estratégia competitiva: técnicas para a análise da indústria e da concorrência. 17. ed. Rio de Janeiro: Campus, 1986.

PRESCOTT, J. E.; MILLER, S. H. Inteligência competitiva na prática. Rio de Janeiro: Campus, 2002.

PRESCOTT, J. E. The evolution of Competitive Intelligence: designing a process for action. Journal of the Association of Proposal Management Professionals, USA, p. 37-52, Spr., 1999.

REDEPETRO RN. Disponível em: <http://redepetrorn.com.br//>. Acesso em: 23 jun. 2013.

REDEPETRO. Disponível em: <http://www.redepetrobrasil.org.br/>. Acesso em: 23 jun. 
Revista Ambiente Contábil - ISSN 2176-9036 - UFRN - Natal-RN. v. 8. n. 1, p. 258 - 274, jan./jun. 2016.

2013.

SABHERWAL, R.; FERNANDES, I. B. Business Intelligence. Knowledge Management: Challenges, Solutions and Technologies, Upper Saddle River: Pearson/Prentice Hall, 2010.

SANT'ANNA, A. A. Indústria de petróleo e gás: desempenho recente e desafios futuros. Rio de Janeiro: BNDES, 2010.

TARAPANOFF, K. Inteligência organizacional e competitiva. Brasília: Universidade de Brasília, 2001

TYSON, K. W. M. The complete guide to competitive intelligence. Chicago: Kirk Tyson International, 1998.

VEDDER, R. G.; GUYNES, C. S. A study of competitive intelligence practices in organizations. The Journal of Computer Information Systems, V. 19 N. 2, p. 36-39, 2000.

CIOs' perspective on competitive intelligence. Information Systems Management, V. 19, N.4, p. 49-55, 2002.

WALTERS, B. A.; PRIEM, R. L.; SHOOK, C. L. Small business manager scanning emphases and the dominant logic of the business-level strategy. Journal of Small Business Strategy, V.15 n.2, p. 19-32, 2005.

WIIG, K. M. Knowledge management foundations: thinking about thinking - how people and organizations create, represent, and use knowledge. v.1 Arlington Texas: Schema, 1993.

YAP, C. S.; RASHID, Md Z; SAPUAN, Dewi A. Organizational strategy and competitive intelligence practices in Malaysian public listed companies. Information Research, v. $17 \mathrm{n}$. 4, 2012. 